NOUVELL

\section{Origine ethnique et composition du microbiote intestinal}

Mélanie Deschasaux ${ }^{1}$, Koos Zwinderman ${ }^{1}$, Max Nieuwdorp ${ }^{2-4}$
${ }^{1}$ Department of Clinical Epidemiology, Biostatistics and

Bioinformatics, Amsterdam UMC-Academic medical center,

Meibergdreef 91105 AZ Amsterdam, Pays-Bas.

${ }^{2}$ Amsterdam Diabetes Center, Department of internal

medicine, Amsterdam UMC, Pays-Bas.

${ }^{3}$ Wallenberg laboratory, Sahlgrenska Academy, University of

Gothenburg, Suède.

${ }^{4}$ Department of vascular medicine, Amsterdam UMC-Academic medical center, Pays-Bas.

m.deschasaux@eren.smbh.univ-paris13.fr
$>$ Nos intestins abritent des communautés composées de milliards de microorganismes considérés actuellement comme des acteurs clés de notre santé [1] $(\rightarrow)$. $(\rightarrow)$ Voir le numéro thématique Le microbiote, $\mathrm{m} / \mathrm{s} \mathrm{n}{ }^{\circ} 11$, novembre 2016, pages 919-1037
Comprendre les facteurs qui modulent ces communautés constitue donc un enjeu important. Si la composition du microbiote intestinal est propre à chaque individu, l'influence d'autres paramètres est admise, notamment l'alimentation, le mode de vie, la prise de médicaments, l'environnement, les premières années de vie ou la génétique [2-5], la plupart de ces paramètres étant, dans une certaine mesure, communs aux individus partageant la même origine ethnique. Jusqu'à présent, la majorité des études ayant évalué le lien entre ethnicité et composition du microbiote intestinal ont comparé des groupes de taille limitée, vivant dans des zones géographiques distinctes et avec des modes de vie très différents (traditionnel versus occidental, rural versus urbain) [6]. Pour tenter de discriminer le rôle de l'origine ethnique et celui de l'environnement ou du mode de vie, nous avons donc conduit une étude [7] visant à décrire la composition du microbiote intestinal chez des individus ayant des origines ethniques différentes mais vivant dans une même ville.

Au sein de la cohorte HELIUS (healthy life in an urban setting), notre étude a porté sur 2084 individus résidant à Amsterdam (Pays-Bas) et appartenant à l'un des six groupes ethniques les plus représentés dans la ville [8]: des Néerlandais $(n=439)$ et des immi- grés de $1^{\text {re }}$ ou $2^{\mathrm{e}}$ génération originaires du Suriname (ascendance africaine, $\mathrm{n}=443$ ou sud-asiatique, $\mathrm{n}=358)$, de Turquie $(n=197)$, du Maroc $(n=280)$ et du Ghana $(n=367)$. Lors de leur entrée dans la cohorte, ces participants ont rempli un questionnaire portant sur leurs caractéristiques sociodémographiques, leur mode de vie et leur état de santé, et ont subi des examens clinique et biologique. La composition du microbiote intestinal a été déterminée par le séquençage du gène codant l'ARNr l6S (ARN ribosomique) à partir d'un échantillon de selles.

L'ethnicité s'est révélée contribuer de manière significative aux différences de composition du microbiote intestinal entre les individus (diversité $\beta^{1}$ mesurée par plusieurs indices dont l'indice de Bray-Curtis $^{2}$ ). Sur la Figure 1, on peut en effet observer une plus grande proximité des individus de même ethnicité, suggérant une plus grande similarité de composition de leur microbiote intestinal, même si aucune séparation claire n'a été notée. La seule contribution de l'ethnicité à la diversité $\beta$ était d'environ $6 \%$, ce qui est relativement important par rapport aux 16,4 et $18,7 \%$ de contribution globale de différents déterminants étudiés dans le cadre de deux larges études de cohortes (réalisées récemment sur 1000 personnes en Belgique et aux Pays-Bas) [3, 4].

${ }^{1}$ Elle compare les espèces présentes entre deux milieux donnés.

2' ${ }^{\prime}$ indice de dissimilarité de Bray-Curtis est compris entre 0 (les deux échantillons ont la même composition) à l (les échantillons sont totalement dissemblables).
De même, la diversité $\alpha^{3}$ du microbiote intestinal, représentant pour une personne le nombre de bactéries et leur répartition au sein de différents types bactériens, diffère en fonction des groupes ethniques. Elle est notamment plus élevée chez les Néerlandais et plus faible chez les Surinamais d'ascendance sud-asiatique (Figure 2).

$\varepsilon n$ étudiant différentes caractéristiques individuelles potentiellement sousjacentes aux associations entre ethnicité et microbiote intestinal, nous avons observé qu'aucune n'atteignait individuellement la taille d'effet observée pour l'origine ethnique. L'ethnicité est ainsi restée le facteur le plus déterminant des diversités $\alpha$ et $\beta$ du microbiote intestinal, même après ajustement sur l'ensemble des paramètres étudiés: l'alimentation, le sexe, l'âge, l'éducation, la zone de résidence, l'indice de masse corporelle (IMC), l'alcool, le tabac, l'activité physique, le centre et la période de collecte des selles, le groupe de séquençage.

L'état de santé métabolique, inégal entre les groupes ethniques (par exemple, une prévalence plus importante de diabète chez les Surinamais sud-asiatiques, moindre prévalence d'obésité chez les Néerlandais), et potentiellement associé au microbiote intestinal [2], n'a pas expliqué non plus le lien observé entre origine ethnique et diversités $\alpha$ et $\beta$ (profils ethniques de diversités $\alpha$ et $\beta$ similairement observés chez 646

\footnotetext{
${ }^{3}$ La diversité $\alpha$ est une mesure du nombre d'espèces présentes
} dans un milieu donné. 


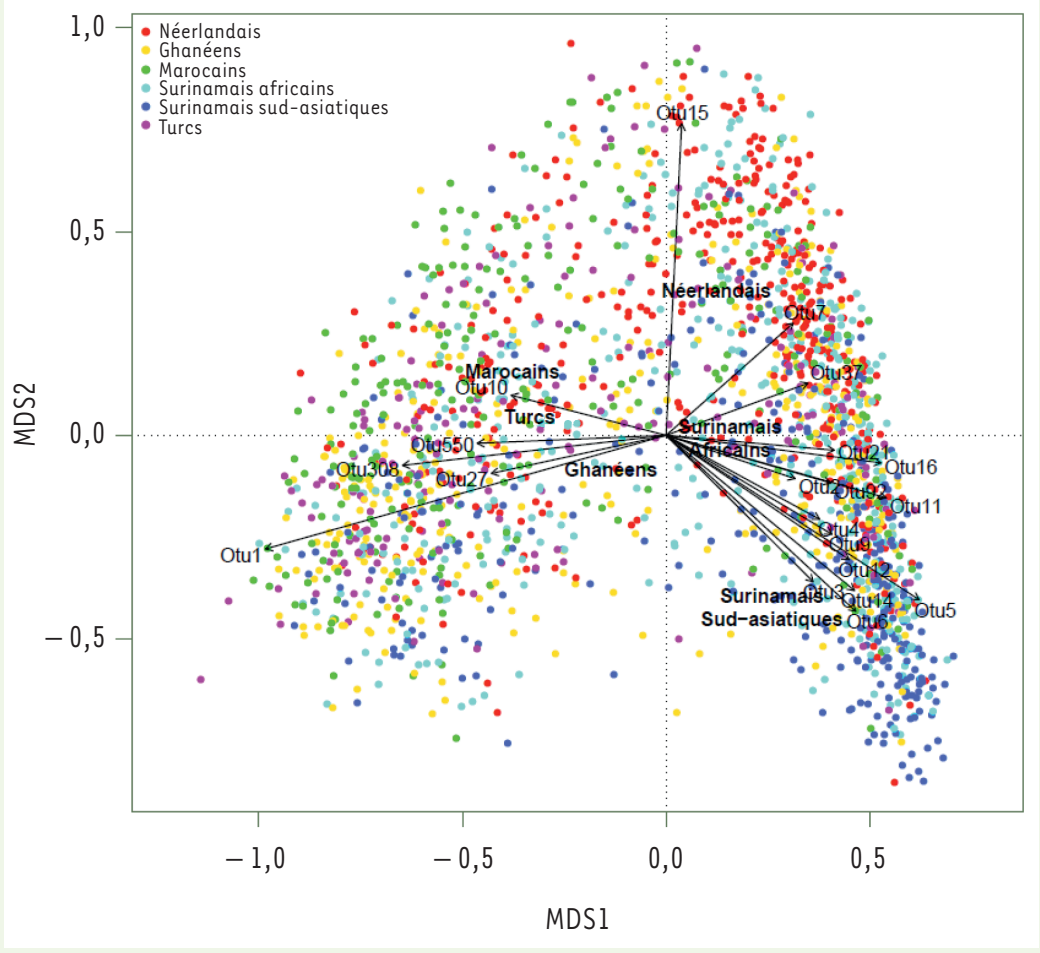

Figure 1. Dissimilarités de composition du microbiote intestinal au sein de 2084 individus issus de la cohorte multi-ethnique HELIUS. Représentation par analyse en coordonnées principales (multidimensional scaling [MDS]) utilisant l'indice de dissimilarité de Bray-Curtis calculé sur les abondances relatives d'OTU (operational taxonomic unit). Chaque point sur la figure correspond à la composition globale du microbiote intestinal pour une personne. L'ethnicité explique 5,7\% des dissimilarités de composition du microbiote intestinal (PERMANOVA ${ }^{*} P=0,001$ ). Le centroïde correspondant à chaque groupe ethnique est représenté par le nom de ce groupe sur la figure. Les 20 OTU contribuant le plus aux dissimilarités de composition entre individus ( $R^{2}$ issus des modèles de PERMANOVA) sont représentés sur la figure. De bas en haut et de gauche à droite: Otul Prevotella copri, Otu27 Prevotella, Otu308 Prevotella copri, Otu550 Prevotella copri, Otu10 Dialister, Otu15 Oscillospira, Otu7 Ruminococcus bromii, Otu37 Coprococcus, Otu21 Coprococcus, Otul6 Bacteroides uniformis, Otu92 Clostridium [Clostridiaceæ], Otull Lachnospiraceæ, Otu2 Fæcalibacterium prausnitzii, Otu4 Bifidobacterium, 0tu9 Blautia, Otu5 Bacteroides, Otul2 Bacteroides, Otul4 Coprococcus, Otu6 Blautia, Otu3 Roseburia Fæcis. Le premier axe d'analyse en coordonnées principales (MDS1) porte 18,4\% de variabilité et le second axe (MDS2) en porte 6,3\%. * La PERMANOVA est l'analyse multivariée de la variance par permutations.

individus sans diabète, syndrome métabolique ou obésité).

L'origine ethnique est associée à la variation de l'abondance relative de 559 OTU (operational taxonomic unit, « espèce » moléculaire) sur les 744 étudiées, avec des différences d'abondance plus marquées chez les Néerlandais et les Marocains (majorité d'OTU enrichies) ainsi que chez les Surinamais sud-asiatiques (majorité d'OTU appauvries), en et plus élevées chez les Surinamais, équivalentes chez les Néerlandais) complétés par une troisième direction associée au type Clostridiales (Oscillospira, Ruminococcus bromii, Coprococcus; plus élevées chez les Néerlandais). Ces 3 taxons caractéristiques, correspondant aux entérotypes [9], ont typiquement été observés dans d'autres populations et sont associés à l'origine ethnique dans cette cohorte.

Notre étude est l'une des rares à avoir comparé la composition du microbiote intestinal entre individus occidentaux et non-occidentaux vivant dans un même environnement. Les précédents résultats montraient généralement une diversité $\alpha$ plus faible dans les populations occidentales par rapport aux populations non-occidentales, mais probablement confondu par le style de vie (urbain occidental versus traditionnel/rural non-occidental). Dans cette étude, les Néerlandais présentaient un microbiote intestinal avec une plus grande diversité $\alpha$, généralement considéré comme plus sain [10], et un enrichissement en de nombreuses OTU, parmi lesquelles un ensemble de type Oscillospira, Christensenellaceae, Mogibacteriaceae et Rikenellaceae, associé à une moindre masse corporelle $[5,11]$ et, à l'inverse, particulièrement appauvri chez les Surinamais sud-asiatiques.

L'ethnicité comprend de nombreux aspects - génétique, habitudes culturelles, migration (par exemple, statut socioéconomique, recours aux soins de santé et aux antibiotiques, milieu de vie lors de l'enfance) - qui peuvent contribuer à façonner le microbiote intestinal de manière spécifique (exposition aux nutriments et aux xénobiotiques, régulation du système immunitaire, colonisation microbienne initiale et ultérieure). Aucune des caractéristiques que nous avons étudiées en tant que potentiels facteurs de confusion majeurs n'a reflété entièrement l'effet de l'ethnicité seule. Ce surplus d'effet peut suggérer l'implication d'autres facteurs que ceux étudiés, notamment 


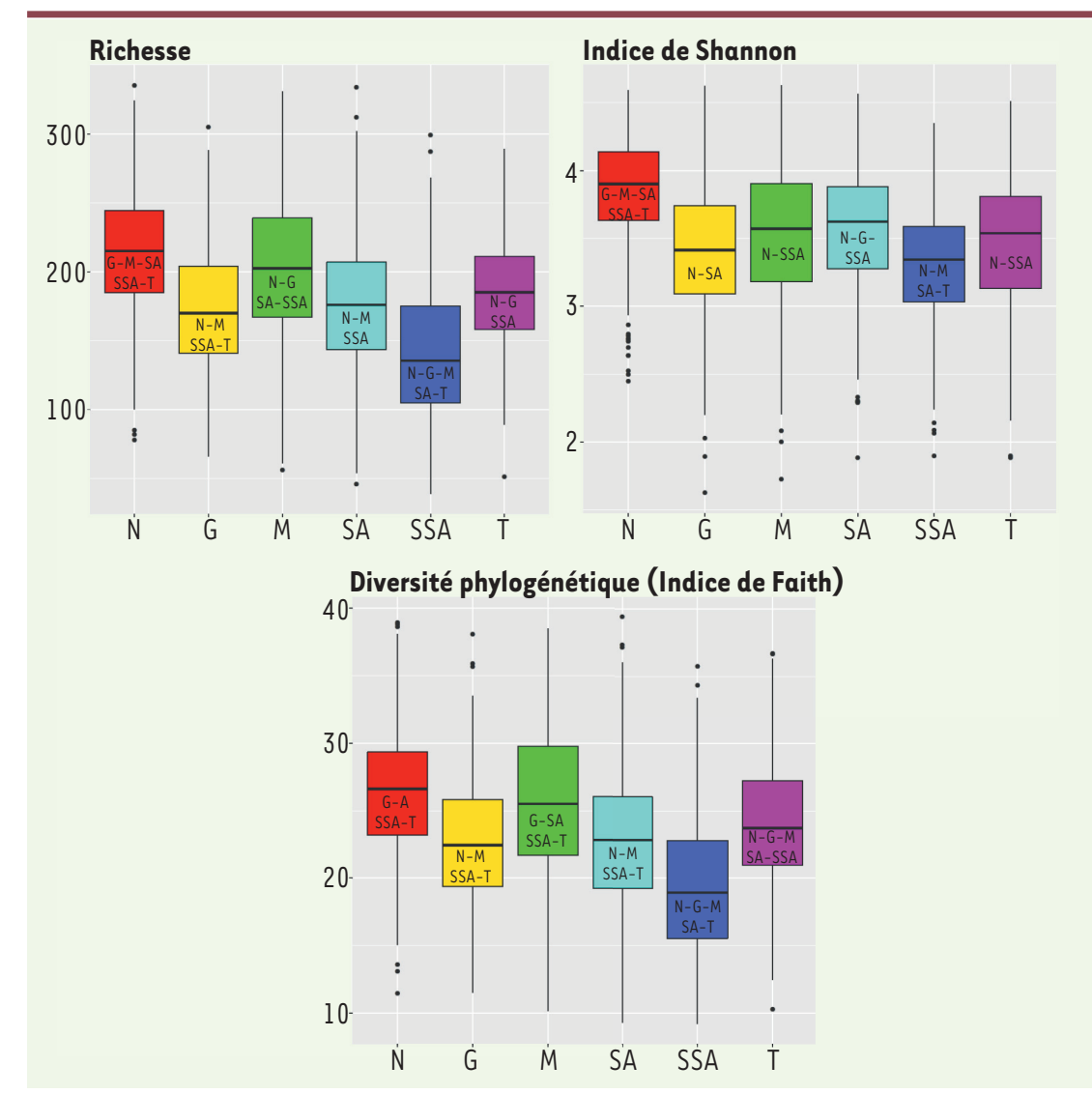

Figure 2. Diversité alpha et origine ethnique au sein de la cohorte HELIUS. Description de la diversité alpha (richesse, indice de Shannon* et indice de diversité phylogénétique de Faith ${ }^{\star \star}$ calculés $^{*}$ au niveau des OTU [operational taxonomic unit] en fonction de l'origine ethnique au sein de la cohorte HELIUS [ $n=2084]$ ). Les boîtes à moustache représentent la médiane (ligne centrale), les quartiles inférieurs et supérieurs (les limites de la boîte), 1,5 X l'intervalle interquartile («moustaches »), et les valeurs marginales (points). Les lettres au sein de chaque boîte indiquent les groupes pour lesquels les différences sont statistiquement significatives ( $t$ tests 2 à 2 , correction de Bonferroni ${ }^{\star \star \star}$ pour tests multiples : $\left.P<3,33 \cdot 10^{-3}\right)$. N : Néerlandais, $G$ : Ghanéens, $M$ : Marocains, SA : Surinamais africains, SSA : Surinamais sud-asiatiques, $T$ : Turcs. *L'indice de Shannon, fondé sur l'entropie, permet de mesurer la diversité spécifique (nombre d'espèces présentes dans un milieu et répartition des individus au sein de ces espèces). ** (et indice utilise les longueurs de

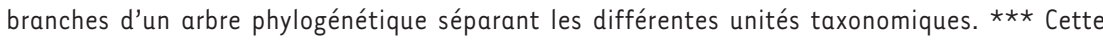
méthode permet de corriger le seuil de significativité lors de comparaisons multiples.

la génétique. Bien que l'influence de la génétique soit considérée comme faible par rapport aux facteurs environnementaux, en particulier l'alimentation [6, 12], elle pourrait néanmoins contribuer à créer divers profils de microbiote intestinal qui seraient affinés par l'environnement [9]. De fait, soutenant une implication de la génétique, nous avons observé des abondances différentielles entre groupes ethniques pour des OTU lactose (persistance de la lactase, un trait déterminé par une mutation du gène $L C T$, sélectionné dans les communautés traditionnellement consommatrices de lait) et la consommation de produits laitiers [13]. Une moindre abondance de Bifidobacterium, utilisant le lactose comme substrat, serait alors liée à de plus faibles quantités de lactose atteignant le côlon après consommation de produits laitiers dans le cas d'une persistance de la lactase (chez les Néerlandais) par rapport à une non-persistance.

Différents profils de microbiote intestinal peuvent refléter des adaptations spécifiques aux conditions environnementales. La plupart des participants non-néerlandais de notre étude étaient des immigrés de première génération arrivés aux Pays-Bas à l'âge adulte et depuis plus de 15 ans. Tout en ayant vécu dans un même environnement pendant une longue période, les participants présentaient néanmoins des profils de microbiote intestinal liés à leur origine ethnique, reflétant probablement une composition acquise avant leur immigration. Les changements accompagnant la migration (par exemple l'offre et la consommation alimentaire, le mode de vie, l'environnement bactérien et chimique) n'ont ainsi peut-être pas induit des perturbations suffisamment importantes face à la résilience du microbiote intestinal, ce qui pourrait conduire à un manque d'adaptation à l'environnement.

Ainsi, nos résultats montrent qu'au sein d'une même population urbaine, l'origine ethnique des individus pourrait être un marqueur de différences de composition du microbiote fécal. L'ethnicité pourrait donc être un facteur important à prendre en compte dans de futures études portant sur le lien entre profils du microbiote et santé (notamment lors de la conception d'interventions), en particulier compte tenu des disparités de santé observées entre groupes ethniques. $\diamond$ How the gut microbiota composition relates to the ethnic background 


\section{REMERCIEMENTS}

L'étude HELIUS est conduite et financée par l'Amsterdam UMC - AMC et le service de santé publique d'Amsterdam. Elle a également reçu des financements de la Dutch Heart Foundation, l'Organisation néerlandaise pour la recherche sur la santé et le développement (ZonMw), de l'Union européenne (FP-7) et du Fonds européen pour l'intégration des immigrés de pays non membres de l'UE (EIF). L'étude présentée ici était en outre financée par des subventions du consortium Le Ducq (17CVD01), du consortium JPI-HDHL MICRODIET et de la Fondation Novo Nordisk (Gut-MMM). M. Nieuwdorp est soutenu par une subvention personnelle ZONMW-VIDI 2013 (016.146.327), et une subvention pour les jeunes talents CVON IN CONTROL de la Dutch Heart Foundation (2013).

\section{LIENS D'INTÉRÊT}

M. Nieuwdorp siège au conseil scientifique de Caelus Health, Pays-Bas. Cette activité n'est pas directement liée à l'étude présentée ici. Aucun brevet, produit en développement ou commercialisé n'est à déclarer.

M. Deschasaux, K. Zwinderman et M. Nieuwdorp déclarent n'avoir aucun lien d'intérêt concernant les données publiées dans cet article.

\section{RÉFÉRENCES}

1. Gilgenkrantz H, Teillaud JL (coordination). Le microbiote : cet inconnu qui réside en nous. Med Sci (Paris) $2016 ; 32: 919-1037$

2. Lynch SV, Pedersen 0 . The human intestinal microbiome in health and disease. $N$ Engl J Med 2016 ; $375: 2369-79$.

3. Zhernakova A, Kurilshikov A, Bonder MJ, et al. Population-based metagenomics analysis reveals markers for gut microbiome composition and diversity. Science 2016 ; 352 : 565-9.

4. Falony G, Joossens M, Vieira-Silva S, et al. Populationlevel analysis of gut microbiome variation. Science 2016 ; $352: 560-4$.

5. Goodrich JK, Waters JL, Poole AC, et al. Human genetics shape the gut microbiome. Cell $2014 ; 159$ : 789-99.

6. Gupta VK, Paul S, Dutta C. Geography, ethnicity or subsistence-specific variations in human microbiome composition and diversity. Front Microbiol 2017 ; 8 : 1162.

7. Deschasaux M, Bouter KE, Prodan A, et al. Depicting the composition of gut microbiota in a population with varied ethnic origins but shared geography. Nat Med 2018; 24 : 1526-31.

8. Snijder MB, Galenkamp H, Prins M, et al. Cohort profile: the healthy life in an urban setting (HELIUS) study in Amsterdam, The Netherlands. BMJ Open 2017 ; $7: e 017873$.

9. Arumugam M, Raes J, Pelletier $\varepsilon$, et al. Enterotypes of the human gut microbiome. Nature 2011 ; 473 : 174-80.
10. Sonnenburg JL, Bäckhed F. Diet-microbiota interactions as moderators of human metabolism. Nature $2016 ; 535: 56-64$

11. Oki K, Toyama M, Banno T, et al. Comprehensive analysis of the fecal microbiota of healthy Japanese adults reveals a new bacterial lineage associated with a phenotype characterized by a high frequency of bowel movements and a lean body type. BMC Microbiol 2016; $16: 284$

12. Rothschild D, Weissbrod 0 , Barkan $\varepsilon$, et al. Environment dominates over host genetics in shaping human gut microbiota. Nature 2018 ; 555 : 210-5

13. Goodrich JK, Davenport \&R, Beaumont M, et al. Genetic determinants of the gut microbiome in UK twins. Cell Host Microbe 2016 ; 19 : 731-43.
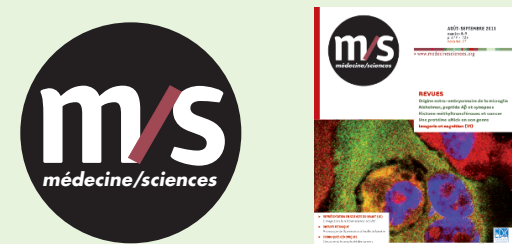

Abonnez-vous à médecine/sciences

Bulletin d'abonnement page 486 dans ce numéro de $\mathrm{m} / \mathrm{s}$

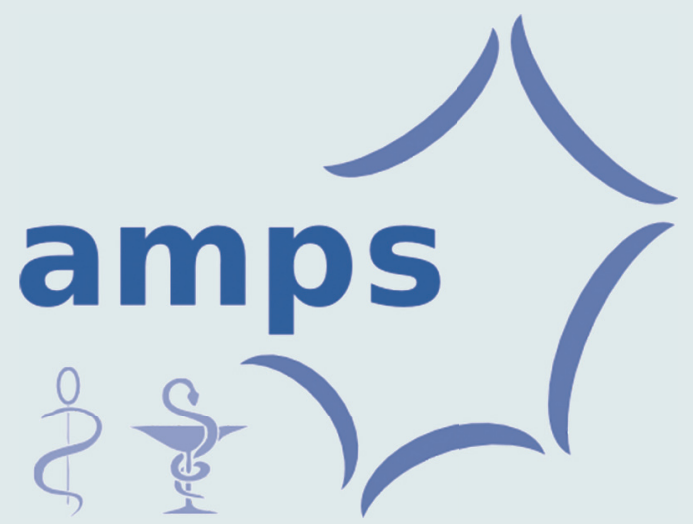

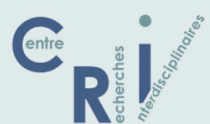

Créée en 2009,

l’Association Médecine/Pharmacie Sciences

(AMPS) a pour objectif principal de rassembler les étudiant(e)s

des double cursus

médecine-sciences

et pharmacie-sciences de France

L'AMPS encourage les approches multidisciplinaires et permet aux étudiants des différentes facultés, ayant des compétences différentes, d'échanger leurs idées et d'interagir entre eux, via un groupe virtuel

(sur les réseaux sociaux) performant, des dîners doubles cursus mensuels et un congrès annuel.

Nous comptons parmi nos membres des étudiants en master, des doctorants, des internes et des cliniciens. Cette formidable diversité permet de mettre en commun les différentes expertises scientifiques et cliniques.

Elle permet également aux plus jeunes de bénéficier des conseils précieux de leurs aînés.

La newsletter, envoyée à tous les membres chaque mois, est un outil que chacun utilise au mieux.

\section{http://www.amps-asso.fr}

\section{Groupe facebook: AMPS (Association Médecine Pharmacie Sciences)}

Sur Twitter : @AssoAMPS 